\title{
Revealing the Phenomena of Heat and Photon Energy on Dealing Matter at Atomic Level
}

\section{Mubarak Ali}

Department of Physics, COMSATS Institute of Information Technology, 45550 Islamabad, PAKISTAN, E-mail: mubarak74@comsats.edu.pk or mubarak74@mail.com

\begin{abstract}
Technology is in the way to reach in its climax but the basic understanding of science in many phenomena is still awaited even though the nature witnesses it. Scientific research reveals strong analogy between photon and electron. Atoms of neutral state when at ground level levitate electron of lateral surface at back surface while placing the bit energy at front surface. Gravitation behavior of that electron starts at centre of relaxation point by including the force of side pole where the pulling force of nearby unfilled state of that atom from the front surface results into depict forcing energy shape-like Gaussian distribution symbol with both ends turned called unit photon. The inertia is being involved at each stage of changing position of that electron by introducing the disappearances of forces of two poles resisting against appearances of forces of two opposite poles when rest to motion and motion to rest in the levitating period. The same is the case in the gravitating period of that electron when rest to motion and motion to rest but under different introducing the disappearances of forces of two poles resisting against appearances of forces of two opposite poles. However, in the stage of levitating or gravitating electron only one force is being involved at one time where opposite force is disappeared. The uninterrupted confined inter-state electrondynamics of neutral state atom while dealing several bits of bit energy having two different shapes result into generate forcing energy shape like a wave. Two bits of bit energy where shape of bit is like opposite integral symbol are being placed along the trajectory of inter-state electron-dynamics during forward-direction cycle and two bits of bit energy where shape of bit like integral symbol are being placed along the trajectory of inter-state electron-dynamics during back-direction cycle. Generating forcing energy
\end{abstract}


of unit photon in each cycle is pushing to rear side by remaining connected till electron is not restoring the state of rest. Silicon atom is considered as a model system of it. Uninterrupted confined inter-state electron-dynamics result into generate forcing energy that can travel immeasurable length and unavailability of necessitating bit energy at any interrupted stage resulting into generate an overt photon. Inter-state electron-dynamics for at least two cycles generate an overt photon -a photon length twice to unit photon. Under certain interaction of unit photon, it divides into two bits of bit energy instead tits and bits of heat. The mechanism of generating photon characteristic current in silicon atom validates that atoms of various elements are two-dimensional elliptical (circular) discs under the neutral state of their electrons. An isolated electron is being grounded under directed forcing energy to impinge a neutral state atom where it gained instantaneous velocity under sinked (merged) energy resulting into transfer it to elongate atom along east west poles. Matter changes the role of energy and force under various sorts of interactions. Here, heat energy and photon energy explore matter at atomic and electron levels, thus, devise science to describe.

Keywords: heat energy; photon energy; forces of poles; nanoscale phenomenon; atomic scale phenomenon; electron scale phenomenon

\section{INTRODUCTION:}

Humanity is being benefited by heat energy and photon energy since the existence, and electrical phenomena have been also studied since long. Catching fire in various stuffs is a usual phenomenon known since the existence of life. Everyone is taking benefit of these blessings but the understanding behind these phenomena is not only clear but their dealing to various sorts of matter is also unclear.

Many studies are available in the literature dealing with light-matter interaction and it has been covered largely under a phenomenon, namely, surface plasmons. Origin of plasmons was explored in some early published reports [1-4]. A plasmon is a quantum of plasma oscillation and represents the collective oscillations of the free electron gas density - a general definition extracted from the literature. 
The interaction of light (photon) to matter is recognized in the form of various terminologies, namely, phonon, excitons, and plasmon, etc. Recently published review discusses the light-matter interaction by considering the properties of polaritons modes in two-dimensional materials and applications in the certain range of spectrum [5]. The concept of excitons (electron-hole pairs) was first proposed by Frenkel [6] and deals with excited state of an atom in a lattice travelling in particle-like fashion without net transfer of charge. Excitons can be formed on absorption of photon by a semiconductor (quantum dot) [7]; a phonon is a collective excitation in a periodic, elastic arrangement of atoms or molecules in condensed matter.

A vast number of studies deal the formation process of tiny particles -structure evolution at nanoscale. The tiny-sized cluster is a simple chemical compound which has a variety of important applications in diversified areas [8]. The unique nature of nanocrystals solicits fabrication of new materials of controlled features [9]. The likely development of nanoparticles technology is an obvious long-term benefit [10]. With the success of assembling colloidal matter in a useful structure, the atoms and molecules will be treated as materials for tomorrow [11]. The investigation of the dynamics of an individual nanoparticle should be taken as a prime concern prior to go for further solid deliberations [12]. A good understanding on the surface features of nanoparticles will lead the development of higher order materials [13]. Tiny-sized clusters possess molecular-like electronic structures and non-fcc geometric structures [14]. Chemical properties of gold nanoparticles change with size [15].

It has been suggested that localized dynamics of the process is one of the cause that helps to evolve the structure of tiny particles in gold [16-20], silver [20], and carbon [21, 22]. Under the process of synergy or impinging electron streams from the regular external source, electron states of the atoms are being stretched and reveal different levels while they remained bonded in the tiny particles [16]. However, where impinging of electron streams is regular from the connected external source, the underlying atoms of tiny particles show orientational-based stretching of electron states [16]. The stretching of electron states of atoms while occupying tiny particles can be orientationalbased under the process of synergy too [16]. When the stretching of electron states of 
an atom is orientational based, it is said to be the elongation of atom and when it is nonorientational based, it is said to be the deformation of atom and all those atoms of solid state behavior with at least one unfilled electron state reveal such behaviors if they are dealing various sorts of interactions [16-24].

Sir Isaac Newton explained the gravity called Newtonian Physics. There is no such concept of the opposite of gravity in the Theory of General Relativity as explained by Sir Albert Einstein. Bohr proposed that electrons move around the nucleus in allocated orbits where they have fixed energy if atom is in ground state. Therefore, the behavior of levitation, neutral and gravitation dealing by the electrons in their atoms of various nature remains beyond the understandings and the concept of band gap, valence band and conduction band dominated all along through with shells and orbital configurations, thus, was kept away to consider and think on forcing energy and force directed phenomena dealing matter at all scale. A parallel study will discuss the force-directed phenomenon in atoms of various elements while dealing different states [25]. Huge efforts were applied in exploring technological advances and breakthroughs but the efforts that were put forth toward basic and fundamental sciences along with continuity in the explored ones remained in less focus.

In this work, the phenomena of heat energy and photon energy is discussed by considering silicon atom where a bit energy configures along the trajectory of electron while in levitation behavior and a bit energy configures along the trajectory of electron while in gravitation behavior, thus, generating unit photon under one cycle inter-state electron-dynamics. Here, charisma of an atom while at neutral state configuring bits of bit energy into photon energy under confined inter-state electron-dynamics is discussed. The analogy between photon and electron is also explained here.

\section{RESULTS AND DISCUSSION:}

Under the application of photonic current, inert gas atoms split into electron streams [24]; electron streams are being utilized to impinge underlying atoms of tiny-sized particle resulting into deform or elongate them depending on the mechanism of impingement as discussed in the case of bound atom in tiny-sized particle of two- 
dimensional structure. If the impinging of electron streams to underlying atoms of monolayer tiny particle is not in accordance or the structure of tiny particle is not in twodimension, the stretching of electron states remains non-orientational based where deformation of the atoms takes place. Therefore, where atoms deform instead of elongating, their structure don't modify to structure of smooth elements. A tiny shaped particle of gold was taken as a model system to explain the mechanism of elongation along with the modification of structure to structure of smooth elements where elongated triangular-shaped tiny particle reveals localized gravity at single point [23].

Energy should be configured (placed) in the form of forcing element to travel or propagate it from one location to another either through the travelling medium or through the propagating medium. Thus, the smallest entity which can deal the force and energy together, both in its travelling or propagating, is the unit photon. The word propagation sounds better when it is dealing the inter-state electron gap in atoms of various elements while the word travelling sounds better other than dealing the interstate electron gap where unit photon is dealing the air (normal) medium and others. Inter-state electron gap of atoms sounds better when dealing a neutral state of an atom. Many ground state atoms deal their neutral state as well, in many ways, as discussed elsewhere [25]. Thus, a unit photon should constitute shape-like Gaussian distribution symbol with both ends turned upward or in the case of inverted Gaussian distribution symbol with both ends turned downward as shown in (1) of Figure 1. Interaction of unit photon to certain medium under suitable scheme results into its division into two equal parts (shape like integral symbol and shape like opposite integral symbol) called a bit energy in either case as shown in (2) and (3) of Figure 1, respectively. Each one of them deals a bit energy along the trajectory of inter-state electron-dynamics in certain atom of neutral state at ground level as discussed below. The heat energy is generated when a photon of certain length even a 'unit photon' divides under the interaction of certain medium. On interaction of unit photon to solid medium like an electron when resulted into parallel compressing of both arms starting from the turned ends where instead of division, it deals sinked (merged) form of energy as shown in (4) of Figure 1, which is purely related to heat. On interaction of unit photon to certain medium where 
instead of dividing into two parts or transforming into sinked (merged) energy, they divide into several parts or its divided two parts (related to bit energy) further divide into parts, they are also related to heat energy but, in the parts where their pieces involved the regions of unit photon dealing to node and antinodes, they are still forcing entities. Nevertheless, in the case where the arms of unit photon neither compressed nor divided into two parts (two bits of bit energy) while undertaking interaction (s) to certain medium, it divided into several parts as shown in (5) of Figure 1 and they are related to tits and bits of heat. Those tits and bits dissipated in the mediums like liquid solutions and other suitable mediums to heat them uniformly, thus, obeying the first law of thermodynamics. The tits and bits can be the source of forcing energy while placing along configuring trajectory of executing inter-state electron-dynamics of a neutral state atom as discussed below.

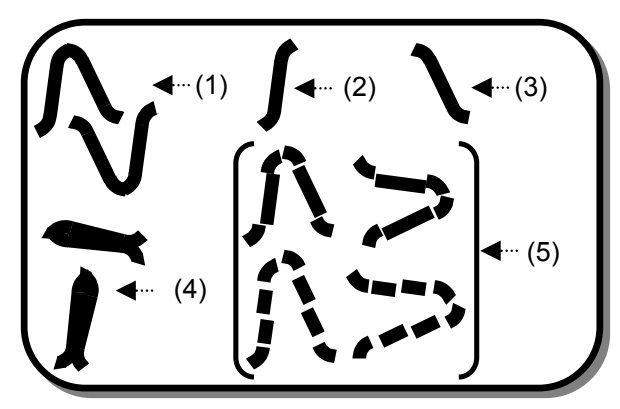

Figure 1: (1) The smallest entity of forcing energy shape-like Gaussian distribution symbol with both ends turned called unit photon, division of unit photon in shape like (2) integral symbol and (3) opposite integral symbol, (4) sink (merge) energy of unit photon and (5) division of unit photon into several schemes of tits and bits of heat.

On interacting photons of longer wavelengths to water, they raise the temperature of the medium to greater volume but under less degree of hotness and vice versa. Similarly, long length photon (overt photon) carries more energy as compared to the one called unit photon. As the heat entities of interacted photons under certain interaction to different sorts of medium deal localized energy at large extent, thus, they can't deal the force influencing a body from the distance; the element of force diminished to large extent because of their broken regions belonging to nodes and antinodes in their previously known photon as well. Such heat entities are being absorbed along with their dissipation in the medium like water or solutions of different 
composition or other suitable mediums not working as an insulator. The term 'partially propagate' may be employed as the divided pieces of unit photon are not completely still and their tits and bits (along with force energy was configured due to inertial property of the electron) are still in the propagation mode to inter-state electron gap of water molecules and associated containments (components) where in addition to conduct heat, the medium also starts conducting the current. Due to that phenomenon, a body often faces the electric shock, a shock which is due to photonic current, on connection of wire to liquid medium dealing propagation of photonic current.

A photon characteristic current, in any length, propagates in inter-state electron gap of atoms as they contain both width and length of gap within the amplitude and wavelength of that photon [24]. However, it is observable that the burst or damage of copper or steel wire in the case of halting propagation of photons characteristic current was either due to distorted (out of order) inter-state electron gap or exceeded density propagation of photons. The length of photon depends on the number of cycles counting at the point of generating source until the interruption takes place. If the confined electron-dynamics remain operational and consistent while retaining within inter-state gap, it remains uninterrupted resulting into generate forcing energy under each connecting cycle shape like a wave as shown in (1) of Figure 2; it is also called an overt photon where the interstate electron-dynamics configured forcing energy after execution of six cycles (three in the forward-direction and three in the back-direction). Under appropriate coinciding to a medium, an overt photon is converted into different number of bit energy depending on the length of overt photon where each connected unit photon divided into two bits of bit energy. As overt photon is the multiple of a unit photon as shown in (1) of Figure 2. Due to its interaction to medium, it converted into several number of bit energy. When it was interacted to medium where angle of its generating point in space (travelling) and angle of the medium at ground level to which interacted is the same as shown in (2) of Figure 2 , it divided into twelve bits of bit energy as shown in (3) of Figure 2. Such bits of bit energy are being absorbed by the silicon lattice on penetrating through the laminated glass surface, thus, working again as photon energy as discussed below. On the other hand, when it was interacted normal to plane (surface of the suitable medium) as shown 
in (4) of Figure (4), it divided into tits and bits as shown in (5) of Figure 2. However, as discussed in the case of Figure 1, bit energy further divides into tits and bits of heat if it is not timely utilized. It can be deduced that an overt photon where several unit photons combined, on dividing further under miscellaneous interactions, it converts into tits bits of heat where they contain scattered energy dissipating uniformly in the medium like water, thus, obeying the first law of thermodynamics.

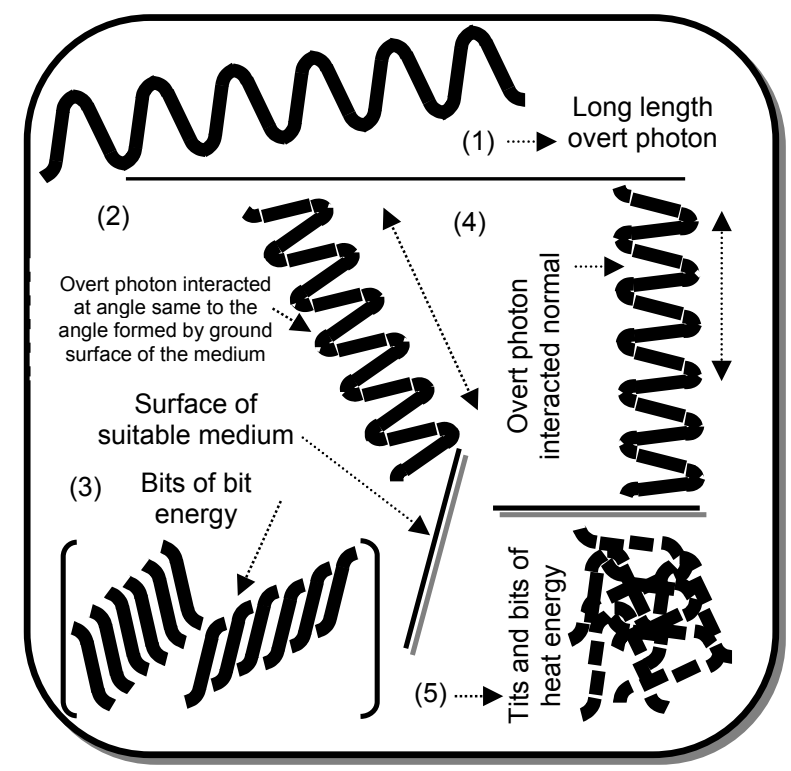

Figure 2: (1) An overt photon generated under the trajectory of three forward-back uninterrupted cycles of inter-state electron-dynamics, (2) interaction of overt photon to suitable glass medium when its angle in space is equal to forming angle of glass plane placed at ground level, (3) formation of several bits of bit energy when incidence angle of overt photon is the same at which glass surface placed at ground level, (4) interaction of overt photon perpendicular to horizontal surface of certain medium at ground level and (5) formation of several tits and bits of heat on interaction of overt photon perpendicular to horizontal surface of certain medium at ground level.

In the case of bits of bit energy, if they don't divide further under the medium compression, they become the complete recipe of a levitating electron at its start (inertia) to at its end (inertia) when shape like opposite integral symbol. But, on placement of bit energy along configured trajectory of levitated period of electron, its shape looks like integral symbol. On connecting another bit energy to back surface to configured energy at centre edge (midpoint) where mainly placing along the configuring trajectory of that electron in gravitating period at front side resulting into generate a unit 
photon shape-like Gaussian distribution symbol with both ends turned. An overt photon contained the forcing energy of at least two-unit photons; thus, it involves at least two nodes and two antinodes.

When neutral state silicon atom deals inter-state electron-dynamics, the relevant electron is being levitated south to north while starting the motion against its inertia and ending the motion against its inertia where a bit energy is being placed along its configuring trajectory. A lateral surface electron of the silicon atom when at neutral state is shown in Figure 3 (a) along with positions of the poles. A neutral state of an atom infers where all occupied electrons are in their states without undertaking any stretching through their energy knots as discussed elsewhere [25]. At neutral state, a silicon atom deals its crystallized state of electronic structure where it behaves two-dimensionally as shown in Figure 3 (a), thus, self-controlling inter-state electron-dynamics purely under existing natural force from four poles. In this context, each electron of the silicon atom while at neutral state deals and undertakes its own force without interfering in force of other.

In Figure 3 (b), a generation of unit photon is shown where, at the centre of relaxation point, a placed bit energy along configured trajectory of the electron is ending when the ended inertia of levitated electron is observed but at the same point another bit energy having the same shape (shape like opposite integral symbol) is being placed which is ending when the ended inertia of gravitated electron is observed. This unit photon is the forcing energy resulted under only one forward-direction cycle of inter-state electrondynamics. In levitation of an electron from the filled state of a neutral state atom, the energy configured along the trajectory of electron was mainly due to steady-state levitation behavior. In gravitation of an electron from the centre of relaxation point, the energy configured under the trajectory of electron was mainly due to steady-state gravitation behavior. That electron included its inertia at the start, in between the trajectory of steady-state levitation behavior and steady-state gravitation behavior, and prior to come at rest. This is a complete cycle of inter-state electron-dynamics in forward-direction as shown in Figure 3 (b). At filled state electron of lateral surface in silicon atom, a bit energy enters from the top side of front surface resulting into 
disappearances of paired forces (north west forces), as a result, that electron removes from the state that is being clamped by its energy knot under the appearances of paired forces (south east forces). It levitates under steady-state behavior on dealing inertia where the force of south pole only remains operational under its steady-state behavior as shown in left-side of Figure 3 (b). At the point of start removing electron from clamped energy knot and prior to dealing steady-state behavior of levitation, the energy is being placed along configured trajectory of electron is due to its inertia. Therefore, a bit energy entered from the front surface of the atom is mainly placed at back surface along configuring trajectory of levitating electron and only a small portion of energy is placed along the trajectory configured due to its inertia. Another bit energy is being entered from the back surface of centre of relaxation point shape like ring where connected the top position to the ending position of first configured bit energy. However, that bit energy entered from the back surface of the atom is mainly placed at front surface along configured trajectory of gravitating electron and only a small portion of placed energy is along the centre edge of back surface as shown in right-side of Figure 3 (b).

The energy configured along the trajectory where electron changed the state (rest to motion or motion to rest) was due to its inertia while the energy configured under the trajectory in steady-state behaviors of electron was due to its levitation and gravitation behaviors. The process of inter-state electron-dynamics in neutral state ground level atom on completing six cycles (three in the forward-direction and three in the backdirection) shaped the twelve bits of bit energy like a wave where six of them have the shape like opposite integral symbol and six of them have the shape like integral symbol as shown in Figure 3 (c); a bit energy shape like opposite integral symbol is working for forward cycle of inter-state electron-dynamics, whereas, a bit energy shape like integral symbol is working for back cycle. Entering of bit energy from the front side but mainly configuring/placing along the back side following by the entering of another same shape bit energy from the back-side relaxation point at centre but mainly configuring along the front side in the forward-direction cycle and vice versa for back-direction cycle pushing itself to rear space by remaining connected to electron of generating forcing energy if it 
continued executing under inter-state dynamics. This is a forcing energy which is related to photon energy. Because of long length of this photon as compared to unit photon, it is termed as overt photon. The generating forcing energy interrupted at certain point, a new forcing energy of photon starts generating. The same process takes place in many atoms of the lattice which results in generating photonic current under some additional modification required for a silicon solar cell. Each cycle of inter-state electron-dynamics generates forcing energy of unit photon shape-like Gaussian distribution symbol with both ends turned where two bits of bit energy is being placed along the configured trajectory. The continuous supply of bit energy to electron will increase the length of forcing energy; hence, longer length of overt photon is resulted on interruption.
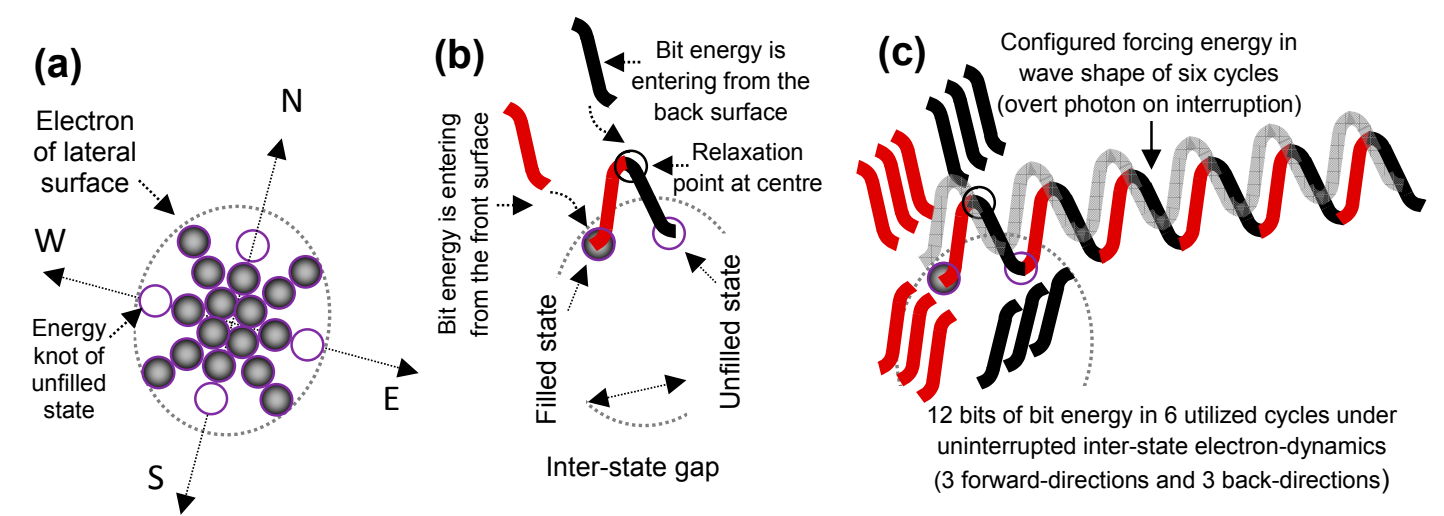

Figure 3: A neutral state silicon atom (a) shows electronic structure, (b) generated forcing energy of unit photon where two bits of bit energy is being utilized and (c) generating overt photon under forcing energy of six cycles inter-state electron-dynamics where total twelve bits of bit energy are being utilized.

In the case where an electron doesn't deal direct usage of the bit energy, the heat energy of divided three portions of a bit energy can be employed along the trajectory resulting into form the bit energy of first half cycle and same is the case of a bit energy for second half cycle resulting into generate unit photon. The overall shape of configured energy along the trajectory of half-cycle during levitation period of electron is like integral symbol $(\delta)$. The overall shape of configured energy along the trajectory of half-cycle during gravitation period of electron is like opposite integral symbol. So, the energy configured in the two shapes of integral symbols, opposite to each other, remained connected under the path of electron resulting into give the overall shape of 
configured energy shape-like Gaussian distribution symbol with both ends turned upward as shown in Figure 4 (a) - a unit photon. The energy configured along the path where an electron is going to levitate under the disturbance of its state of rest is due to its inertia as it is reaching in the state of levitation behavior to relaxation point - (1) to (2) in Figure 4 (a). In the time of levitating electron, the energy configured under the trajectory is due to steady-state levitation behavior - between (2) and (3) in Figure 4 (a). On turning the electron to go into relaxation, the energy configured is due to inertia - (3) ascending curved part to mid-position of the curve in Figure 4 (a). When the electron is turned into gravitation behavior, instead of relaxing at centre edge, energy configures again due to inertia - (4) descending curved part to mid-position of the curve in Figure 4 (a). During gravitating the electron, the energy configured under its trajectory is due to steady-state gravitation behavior - between (4) and (5) in Figure 4 (a). The energy configured on reaching to neutral position of electron is again under the inertia as it changed the state from motion to rest - (5) to (6) in Figure 4 (a). A same amount of inertia is being involved at all stages of changing state of electron; rest to motion, motion to rest, rest to motion and motion to rest. In the trajectories of electron where its inertia involved, the configured (placed) energy deals the nodes and antinodes in propagating or travelling forcing energy (photon or wave). These regions of inertia are responsible to force the energy from one point to another.

(a)

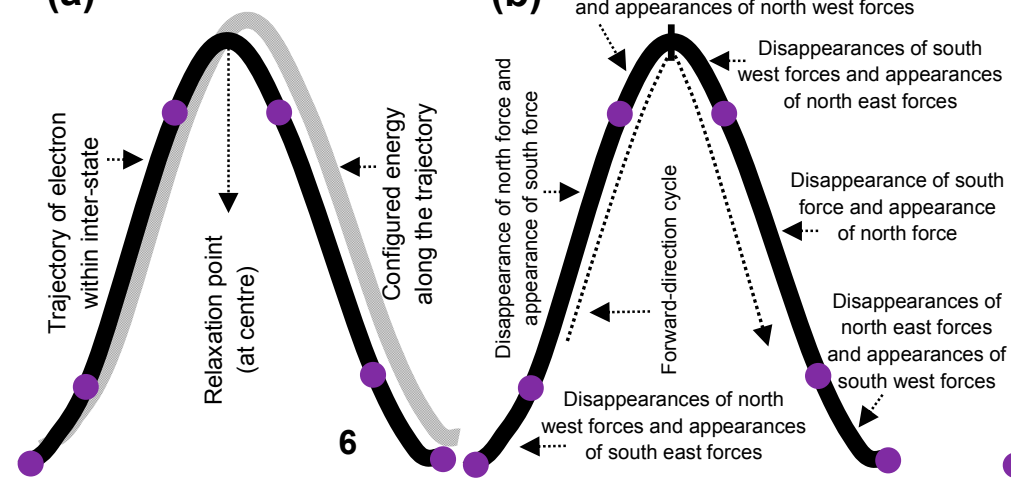

(c) Disappearances of north west forces

(C) and appearances of south east forces

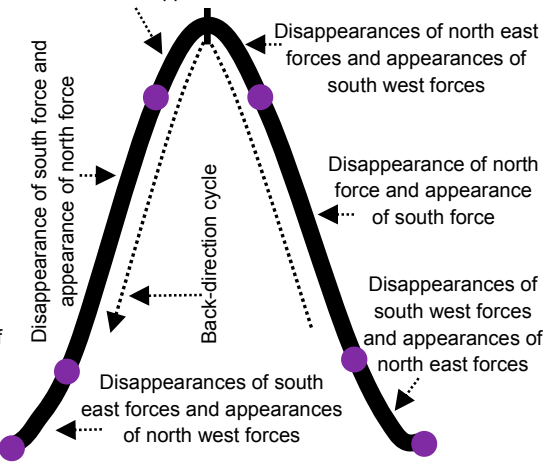

Figure 4: (a) different regions of configured forcing energy of unit photon under confined inter-state electron-dynamics - front side (dark color) shows trajectory of electron and rear side (grey color) shows configured forcing energy of unit photon and (b) disappearances and appearances of different forces at 
different positions of top left-side lateral surface electron in Figure 3 (a) while executing inter-state dynamics for one cycle to (b) forward-direction (left to right) and (c) back-direction (right to left).

The disappearances and appearances of forces at different positions of electron while configuring energy along the trajectory of executed inter-state dynamics for forwarddirection cycle is shown in Figure 4 (b). The disappearances and appearances of forces at different positions of electron while configuring energy along the trajectory of backdirection cycle is shown in Figure 4 (c) where forces at each position are opposite in description.

The element of levitational force influencing is responsible to move electron of target (for left-side lateral surface electron in Figure 3a) south to north side and at inertia to inertia where a bit energy having shape like opposite integral symbol is placed along the trajectory. The element of gravitational force influencing is responsible to move electron of target (for left-side lateral surface electron in Figure $3 a$ ) north to south side and at inertia to inertia where a bit energy shape like opposite integral symbol is placed along the trajectory. The inter-state electron-dynamics remained uninterrupted under the neutral state ground level of the atom. Tits and bits of heat other than 'bit energy' can also configure along the trajectory of electron in the case of either behavior if they are in loop restricting under inter-state electron-dynamics. Therefore, in atoms of silicon lattice interacting photons where dealing one step division on their continuous supply are the source of generating photons characteristic current under fast uninterrupted confined inter-state electron-dynamics. A unit photon when divides into two bits of bit energy, it gives a bit energy shape of opposite integral symbol for half forward-direction cycle and a bit energy shape of integral symbol for half back-direction cycle. Therefore, two-unit photons on dividing into four bits of bit energy will configure the energy of one forwarddirection cycle and one back-direction cycle. It appears that atoms of many materials are eligible to deal this bit energy if succeeding in maintaining their neutral state at ground level.

A photon of forcing energy builds standalone isolated electron to ground for the purpose to impinge the underlying suitable atom of solid state as shown in Figure 5 (a). Interaction of photon to electron results into converting forcing energy to merged (sinked) energy in which altogether force element involved to gain instantaneous 
velocity of the electron as shown in Figure 5 (b). Under instantaneous velocity, that electron transferred sinked energy to underlying neutral state ground level atom as shown in Figure 5 (c). As a result, that atom elongated along east west poles under transferred energy to it, externally, which is shown in Figure 5 (d). The rebounded electron goes toward north side while revealing the levitational force as shown in Figure 5 (e) where it will never ground if it is in the isolated state. The electron is being levitated because it is always forbidden to come at any unfilled state of any atom where forces are protecting it to be ion since the birth and further detail is given elsewhere [24].

(a)

(b)

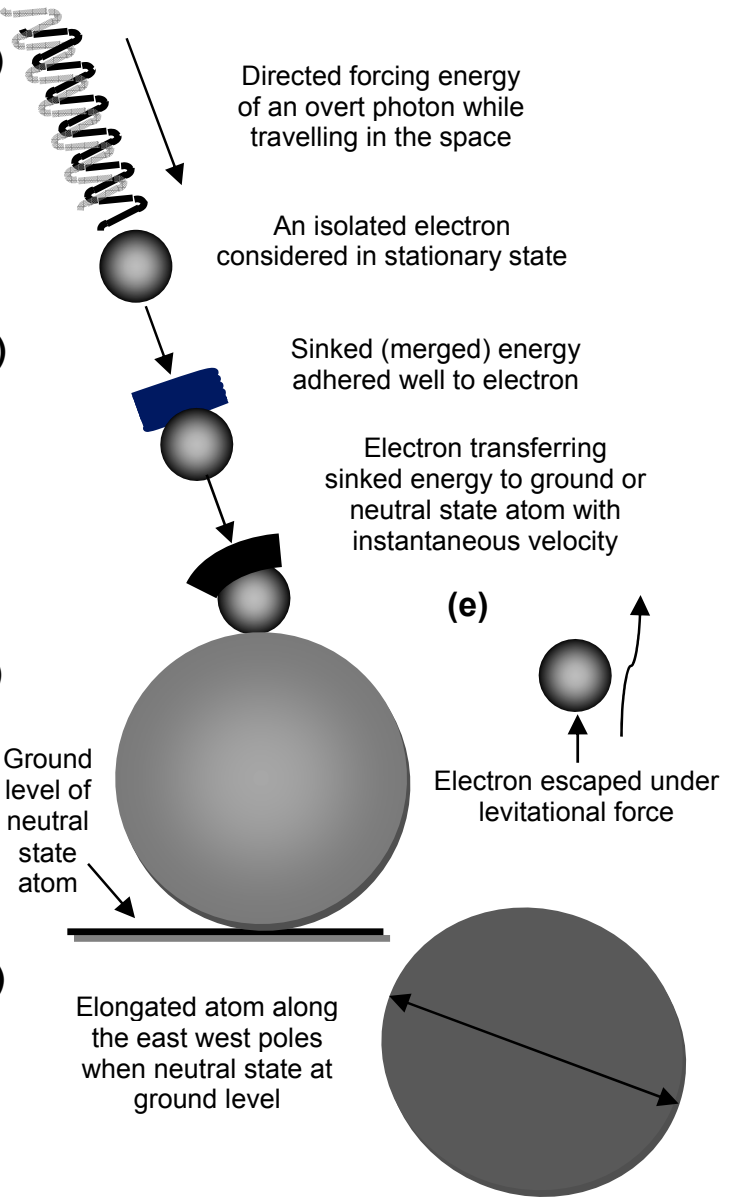

Figure 5: (a) a directed overt photon is in the state of interaction to the isolated electron (considered stationary) under carried discrete forcing energy, (b) overt photon converted into sinked (merged) energy where the element of force is converted into instantaneous velocity of the electron, (c) accelerated electron under instantaneous velocity impinged to the underlying neutral state at ground level atom where it transferred the sinked (merged) energy to the atom while incontact, (d) transferred energy to atom of neutral state, externally, it (that atom) elongated along east west poles, and (e) impinged electron goes alongside on transferring energy to atom depending on the mechanism of its impingement where it is being levitated under force.

In the solar panel where each silicon cell is connected in the series add up the generating number of photons characteristic current under their certain fabrication procedure. Thus, silicon atoms in their solar cell don't require energy either to compensate levitation behavior or to compensate gravitation behavior of electrons to first come into neutral state at ground level. Therefore, the supplied energy in the form 
of bit energy is straight-forwardly placed under their trajectory. The force is being grounded at the backside of solar cell by using the silver paste or through other means and generating photons characteristic current collected from the front surface dealing to sunlight by holding their laminated panels north to south. As observed in solar panel at certain angle with respect to plane oriented to the south will result into an average maximum power generated throughout the year where silicon atoms in the lattice deal several bits of bit energy directly on division under the interaction of their photons to glass sheet where joining of three portions into a bit energy is avoided resulting into bonus power. Therefore, solar panels based on silicon atoms, the probability of levitating electrons in more atoms becomes higher and systematic resulting results into long length photons under uninterrupted cycles of inter-state electron-dynamics, thus, an effective generation of photonic current. The cycles of inter-state electron-dynamics of silicon atoms are non-stop for longer period where on titling silicon solar panel at certain orientation with respect to base results into varying the efficiency which is quite effective in the peak hours of sunlight. When the atoms are not dealing perfect neutral state of ground level despite the fact of peak sun shine a power generation of solar panel may affect. Depositing few layered ordered structure of silicon atoms at suitable substrate may solve the purpose instead of fabricating 300 to $400 \mu \mathrm{m}$ thick solar cell. Employing various dopants and diffusing elements in the fabrication process of silicon solar cell work for the neutral behavior (at ground level) of filled and unfilled states of atoms where energy knots maintain original shape without stretching to any side. Again, incorporating hydrogen atoms to surface of silicon wafer upto few layers work as the spacers for inter-state electron-dynamics of silicon atoms as the electron of lateral surface move from frontside surface to backside surface and backside surface to frontside surface of atom during each cycle, both in forward-direction and backdirection, to generate photons characteristic current.

As discussed elsewhere [21], the enhanced field emission characteristic of 'tiny grains carbon films' was related to modified graphitic phase tiny grains [21]; tiny grains modified electronic structure to structure of smooth elements are the source of enhanced field emission and display panel applications. Photonic current propagates in 
inter-state electron gap as disclosed [24], hence, structure of smooth elements in modified tiny grains deliver enhanced field emission characteristics of carbon films [21]. Atoms of suitable electronic transitions revealing ground state may levitate their electrons resulting into generate a unit photon (or double cycle photon, etc.) and same is the case for atom placed in-contact where generated photons construct firm energy knots between the atoms on their appreciable inter-crossing which is related to forcing energy-directed phenomenon, thus, binding of atoms in many materials is under a forcing energy-directed phenomenon [26]. The same phenomenon is being considered while studying surface enhanced Raman scattering or localized surface Raman spectroscopy phenomena of nanoparticles [27]. Such phenomena are largely studied in the metallic nanoparticles/particles of anisotropic shapes which deliver enhanced field due to the result of many inter-state electron gap of atoms as discussed elsewhere [24].

Distribution of heat energy to disordered structure is in irregular manner. More heat is resulted in structure where atoms are randomly distributed as photons characteristic current are divided into tits and bits of heat rather than their propagation to inter-state electron gap of embedded atoms in the ordered lattice. The collapsed photons in tits and bits of heat can again transform into bits of bit energy, thus, working as the configuring forcing energy in uninterrupted inter-state electron-dynamics of suitable atoms. Similarly, when photons of different energy disrupt the medium (overt photons), they dissipate energy of heat in the form of tits and bits. Photons assign different roles to matter on converting into one form of energy to another depending on the structural motifs and individual nature of constituted atoms. In Bragg's diffraction, amorphous materials don't reveal any specific structure under the interaction of photons wavelength in X-rays where energy of photons mainly converted into heat rather than visualized in the XRD scan. In some cases, photons characteristic current is utilized to split the matter like inert gas atoms and their electrons streams are being utilized to deform or elongate underlying atoms of electronic transitions [24]. Where length of photons is sufficient along with their configured population, they reveal the light glow while travelling at increasing wavelength in the air medium [24]. In the stone's age, catching fire was discovered while striking an embedded stone to another stone under intensive 
acceleration where captured fluke energy at their point of contact converted into 'forcing energy' while exceeding the wavelength in the air medium and on reaching in the visible range revealed the glow of light but many of the photons of 'forcing energy' transformed into tits and bits of heat along with their decreasing energies for revealing the light glow. The set modalities of all sorts of photons depend on the origin of their generation establishing roles set by the manufacturer or expert under dealing to suitable matter. In this context, structural design is crucial in targeting their specific application and many studies are now targeting and exploring structure either which standalone or in relation to other fields of science [28-37]. However, it has been disclosed elsewhere under original line of experiments that all structural motifs are owing to a forcing energydirected phenomenon and a force-directed phenomenon not only in metallic colloids [18] but also in semi-metallic materials [21, 38].

In the forcing energy-directed phenomenon, force transport (forces) the energy from one location to another. However, energy can't force the force as the force is acting from a distance, but a body possesses energy and forcing energy realizes it. As said, a photon is an entity where the energy is being forced from one point to another. It remains confine within inter-state electron gap in atoms of developed wire where it reveals the propagation more like one-dimension. The standalone entity force deals the influencing of a body from a distance. A force which is acting north to south is considered constant with respect to each point located at earth which is not the case when dealing force south to north in a delegate manner as it is always in disturbance, in the both cases, the position of body occupied certain location and surrounding environment of force where force is more related to behavior of an atom under its builtin electron states as discussed elsewhere [25]. All types of photons are the forcing energy entities and they use space either for propagation or for travelling; in propagation, they secure an almost entire energy but, while travelling, they dissipate energy by giving the remarkable applications.

Transition of gas state carbon to diamond state atom is discussed elsewhere [38] where at instant of binding diamond state atoms involved only a force-directed phenomenon. A recent study expressed the implication of tiny particles comprised 
certain nature atoms while using as nanomedicine [39]. Our recent study shows how gravity introducing at unfilled region of developing geometric anisotropic shape deal predictor packing of equilateral triangular-shaped tiny particles [40]. To measure temperature of such materials and in their selective sizes is the integral part to understand science of their different behaviours and some of the studies shed light on it [41-43]. It is possible to measure temperature of atoms, their nanoscale components and at bulk scale as well without the involvement of thermocouples and introducing various gadgets, theories, etc. What is required to determine is the probability of levitating electrons and placement of bit energy under their configured trajectories in atoms of various elements but locate their neutral state at certain level prior to that is vital. As per nature of atoms, the bit energy (or assembling of tits and bits into bit energy) can be varied and then atoms of various elements possess different number of electrons, number of filled states, filled states positions and number of unfilled states, etc.

On interrupting a wave generating at a point of source under confined electrondynamics executing by a suitable atom, it resulted into long length photon called overt photon, on dealing to appropriate medium while placing at certain angle to certain angled ground surface converts into bits of bit energy following by conversion into tits and bits of heat. A unit photon directly gifts the bit energy which may fulfil the requirement of atoms of many materials when in neutral state at their ground level. An electron has appearable mass and it is being stretched through its wrapped energy knot under the deformation or elongation process of its atom. When it is said that an electron is spoiled, it doesn't mean that the atom of that electron is ionized, but it means that the electron occupying atom is either permanent stretched or eroded as discussed elsewhere [24]. In the case of photon, it doesn't have mass and it involves only discrete energy which is forcing from one point to another. In this context, overt photons neither impinged nor stretched and they don't even strike, they interact to certain medium where their forcing energy is being dissipated in the form of tits and bits or bits of bit energy. While propagating in inter-state electron gap, they govern many applications in addition to working only as photonic current. Photons travelling in suitable medium while 
exceeding the wavelength originate several applications. The nature of dealt photon itself is signified as the energy reveals different behavior. Photons wavelength in visible range enable resolution of the image down to $0.2 \mathrm{~mm}$. Photons energy in the wavelength of $\mathrm{X}$-rays propagate through human body and identify the sign of fracture. Photons characteristic current propagate in suitable wire; thus, they are photonic current. Different cameras and devices manipulate the image on screening reflected photons of certain pace at the surface of an object. Structure of certain materials deliver straight-forward application on dealing heat or photon, for example, solutions of various metallic colloids and quantum dots, on interacting light, split light into different colors depending on the refraction and reflection modes along with size and shape of the nanoparticles/particles and 'tiny grains carbon films' give enhance field emission characteristic based on aligned inter-state electron gap in carbon atoms dealing graphite structure. A recent study explained the role of van der Waals interactions in the case of isolated atom by considering the induced dipoles [44] which can be attained when fluctuations of charge density are in wave-like nature [45].

\section{CONCLUSIONS:}

From the top front surface of ground state silicon atom, a bit energy shape like opposite integral symbol is rightly placed at place of configuring trajectory by a levitating electron starting at its filled state and ending at centre of its relaxation point. From the back surface, another bit energy shape like opposite integral symbol is rightly placed at place of configuring trajectory by gravitating electron starting at centre of its relaxation point and ending at centre of occupying unfilled state. Thus, configured energy in the interstate electron-dynamics is the forcing energy called unit photon shape-like Gaussian distribution symbol with both ends turned. Inter-state electron-dynamics of silicon atom configure forcing energy of unit photon in shape-like Gaussian distribution symbol with both ends turned where uninterrupted electron-dynamics configure forcing energy of photon in a wave-like fashion if the necessitating bits of bit energy for each cycle are available. Where tits and bits of heat assemble along the trajectories of inter-state electron-dynamics in the levitation behavior and in the gravitation behavior by keep 
restricting with the loop, two bits of bit energy shape like opposite integral symbol can be formed along the trajectory of electron while completing one cycle of forwarddirection and same is the case for back-direction cycle.

The electron of lateral surface doesn't remain reside in its occupied state on dealing bit energy from the top front surface of the atom where the appearances of forces are not deceiving at atomic level and then at electron level. The electron of top left-side lateral surface in ground state silicon atom remains levitating and gravitating under inter-state dynamics by involving the appearances of paired forces and disappearances of paired forces wherever its inertia is being involved both in forward-direction cycle and back-direction cycle. The electron doesn't stay at occupying unfilled state due to its repeated behavior of appearances of two forces and disappearances of two forces, thus, goes back following the same path where generating forcing energy of unit photon for each cycle is pushing to rear side by remaining connected to electron till it is not restoring state to rest. The appearances and disappearances of forces at different positions of electron while configuring energy in back-direction cycle are opposite in the description to forward direction cycle.

At one side, heat energy is dealing matter at atomic level to be configuring in interstate electron gap and at other side generating forcing energy of photon is converting to again heat energy under certain interaction of matter (medium). When a photon interacts to certain medium, it divides into bits of bit energy following by further division into tits and bits of heat. A unit photon involves two bits of bit energy and an overt photon (long length photon) involves more than two bits of bit energy depending on the length of forcing energy.

A photon has strong analogy to electron in a manner that it propagates in inter-state electron gap working as photonic current where originating all sorts of applications related to force, energy and force energy at terminal end depending on density of propagation, wavelength, etc. A photon characteristic current is a forcing energy preserving the power at maximal while propagating in inter-state electron gap from one point to another. A travelling photon is an entity transporting the energy from one location to another under exceeding wavelength but decreasing energy. Travelling 
photons of space medium form the same angle while interacting to certain medium of plane also dealing the same angle resulting into divide in several bits of bit energy, in the other way of interaction, divide to tits and bits of heat.

An electron is a matter, perhaps, the smallest entity of matter and it is most valuable when it works within natural confinement where it configures heat energy to photon energy. When an isolated stationary electron is directed by the forcing energy (overt photon) to impinge neutral ground state atom, the forcing energy first converted into sinked (merged) energy enabling the impingement of that electron under instantaneous velocity where transferred the transported energy to elongate in-contact atom to east west poles. Clearly, electrons are matter, they occupy space, and they possess mass and impinge (or strike) under provided conditions. Electrons reveal levitation and gravitation behaviors wherever their atoms deal the neutral behavior with respect to forces of poles and silicon atom deals it at ground level under understandable procedure. Photon energy is an assembled entity of tits and bits of heat where the element of force is dominating while heat energy is the divided parts of photon where the element of force is nearly diminished. Therefore, heat energy and photon energy are not the ones impinging or levitating or gravitating.

\section{References}

[1] D. Bohm, D. Pines, A Collective Description of Electron Interactions. I. Magnetic Interactions, Phys. Rev. 1951, 82, 625-634.

[2] D. Pines, D. Bohm, A Collective Description of Electron Interactions: II. Collective vs Individual Particle Aspects of the Interactions, Phys. Rev. 1952, 85, 338-353.

[3] R. H. Ritchie, Plasma Losses by Fast Electrons in Thin Films, Phys. Rev. 1957, 106, 874-881.

[4] D. Bohm, D. Pines, A Collective Description of Electron Interactions: III. Coulomb Interactions in a Degenerate Electron Gas, Phys. Rev. 1957, 92, 609-625.

[5] T. Low, et al., Polaritons in layered two-dimensional materials, Nat. Mater. 2017, 16, 182-194. 
[6] J. Frenkel, On the Transformation of Light into Heat in Solid. I, Phys. Rev. 1931, $37,17-44$.

[7] O. D. D. Couto Jr. et al., Charge control in $\operatorname{lnP} /(G a, I n) P$ single quantum dots embedded in Schottky diodes, Phys. Rev. B 2011, 84, 125301-7.

[8] M. Brust, M. Walker, D. Bethell, D. J. Schiffrin, R. Whyman, Synthesis of Thiolderivatised Gold Nanoparticles in a Two-phase Liquid-Liquid System, J. Chem. Soc., Chem. Commun. 1994, 801-802.

[9] R. L. Whetten, J. T. Khoury, M. M. Alvarez, S. Murthy, I. Vezmar, Z. L. Wang, P. W. Stephens, C. L. Cleveland, W. D. Luedtke, U. Landman, Nanocrystal Gold Molecules, Adv. Mater. 1996, 8, 428-433.

[10] M. Brust, C. J. Kiely, Some recent advances in nanostructure preparation from gold and silver particles: a short topical review, Colloids and Surfaces A: Physicochem. Eng. Aspects 2002, 202, 175-186.

[11] S. C. Glotzer, M. J. Solomon, Anisotropy of building blocks and their assembly into complex structures, Nature Mater. 2007, 6, 557-562.

[12] S. Link, M. A. El-Sayed, Shape and size dependence of radiative, nonradiative and photothermal properties of gold nanocrystals, Int. Rev. Phys. Chem. 2000, 19, 409453.

[13] C. P. Shaw, D. G. Fernig, R. Lévy, Gold nanoparticles as advanced building blocks for nanoscale self-assembled systems, J. Mater. Chem. 2011, 21, 12181-12187.

[14] Y. Negishi, T. Nakazaki, S. Malola, S. Takano, Y. Niihori, W. Kurashige, S. Yamazoe, T. Tsukuda, H. Häkkinen, A Critical Size for Emergence of Nonbulk Electronic and Geometric Structures in Dodecanethiolate-Protected Au Clusters, J. Am. Chem. Soc. 2015, 137, 1206-1212.

[15] A. Moscatelli. Gold nanoparticles: Metallic up to a point, Nature Nanotechnol. 2015, DOI:10.1038/nnano.2015.16.

[16] M. Ali, I -Nan. Lin, The effect of the Electronic Structure, Phase Transition and Localized Dynamics of Atoms in the formation of Tiny Particles of Gold. http://arxiv.org/abs/1604.07144. 
[17] M. Ali, I -N. Lin, Development of gold particles at varying precursor concentration. http://arXiv.org/abs/1604.07508.

[18] M. Ali, I -N. Lin, Controlling morphology-structure of particles at different pulse rate, polarity and effect of photons on structure. http://arxiv.org/abs/1605.04408.

[19] M. Ali, I -N. Lin, Formation of tiny particles and their extended shapes -Origin of physics and chemistry of materials. http://arxiv.org/abs/1605.09123.

[20] M. Ali, I -N. Lin, Tapping opportunity of tiny shaped particles and role of precursor in developing shaped particles. http://arxiv.org/abs/1605.02296.

[21] M. Ali, I -Nan. Lin, Phase transitions and critical phenomena of tiny grains thin films synthesized in microwave plasma chemical vapor deposition and origin of $\mathrm{v} 1$ peak. http://arXiv.org/abs/1604.07152.

[22] M. Ali, M. Ürgen, Switching dynamics of morphology-structure in chemically deposited carbon films -A new insight, Carbon 2017, 122, 653-663.

[23] M. Ali, The study of tiny shaped particle dealing localized gravity at solution surface. http://arxiv.org/abs/1609.08047.

[24] M. Ali, Atoms of electronic transition deform or elongate but do not ionize while inert gas atoms split. http://arxiv.org/abs/1611.05392.

[25] M. Ali, Why some atoms are in gaseous state and some in solid state but carbon work on either side (2017). (Submitted for consideration).

[26] M. Ali, Structure evolution in atoms of solid state dealing electron transitions. http://arxiv.org/abs/1611.01255.

[27] S. Nie, S. R. Emory, Probing Single Molecules and Single Nanoparticles by Surface-Enhanced Raman Scattering, Science 1997, 275, 1102-1106.

[28] J. Zhao, L. Yang, Structure Evolutions and Metallic Transitions in $\ln _{2} \mathrm{Se}_{3}$ Under High Pressure, J. Phys. Chem. C 2014, 118, 5445-5452.

[29] V. N. Manoharan, Colloidal matter: Packing, geometry, and entropy, Science 2015, $349,1253751$.

[30] J. Park, et al., 3D structure of individual nanocrystals in solution by electron microscopy, Science 2015, 349, 290-295. 
[31] D. Jacobsson, et al., Interface dynamics and crystal phase switching in GaAs nanowires. Nature 2016, 531, 317-322.

[32] T. Tuma, A. Pantazi, M. L. Gallo, E. Eleftheriou, Stochastic phase-change neurons. Nature Nanotech. 2016, 11, 693-699.

[33] J. Rensberg, et al., Active Optical Metasurfaces Based on Defect-Engineered Phase-Transition Materials, Nano Lett. 2016, 16, 1050-1055.

[34] Y. Suzuki, G. Cardone, D. Restrepo, P. D. Zavatteri, T. S. Baker, F. A. Tezcan, Selfassembly of coherently dynamic, auxetic, two-dimensional protein crystals, Nature 2016, 533, 369-373.

[35] C. H. J. Evers, J. A. Luiken, P. G. Bolhuis, W. K. Kegel, Self-assembly of microcapsules via colloidal bond hybridization and anisotropy, Nature 2016, 534, 364368.

[36] I. R. Epstein, B. Xu, Reaction-diffusion processes at the nano- and microscales. Nature Nanotech. 2016, 11, 312-319.

[37] A. Azizi, Spontaneous Formation of Atomically Thin Stripes in Transition Metal Dichalcogenide Monolayers, Nano Lett. 2016, 16, 6982-6987.

[38]. M. Ali, M. Ürgen, Deposition Chamber Pressure on the Morphology of Carbon Films and Binding of Carbon Atoms (Submitted for consideration).

[39] M. Ali, Nanoparticles-Photons: Effective or Defective Nanomedicine, J. Nanomed. Res. 2017, 5(6), 00139.

[40] M. Ali, I - N. Lin, C. -J. Yeh, Predictor packing in developing unprecedented shaped colloidal particles (submitted for consideration).

[41] M. Mecklenburg, W. A. Hubbard, E. R. White, R. Dhall, S. B. Cronin, S. Aloni, B. C. Regan, Nanoscale temperature mapping in operating microelectronic devices, Science 2015, 347, 629-632.

[42] L. Ye, D. Hou, X. Zheng, Y. Yan, M. D. Ventra, Local temperatures of stronglycorrelated quantum dots out of equilibrium, Phys. Rev. B 2015, 91, 205106-8.

[43] F. Menges, et al., Temperature mapping of operating nanoscale devices by scanning probe thermometry, Nat. Commun. 2016, 7:10874 doi: 10.1038/ncomms10874. 
[44] S. Kawai, et al., Van der Waals interactions and the limits of isolated atom models at interfaces, Nat. Commun. (2016) DOI: 10.1038/ncomms11559.

[45] A. Ambrosetti, N. Ferri, R. A. DiStasio Jr., A. Tkatchenko, Wavelike charge density fluctuations and van der Waals interactions at the nanoscale, Science 2016, 351, 1171-1176.

Author's biography:

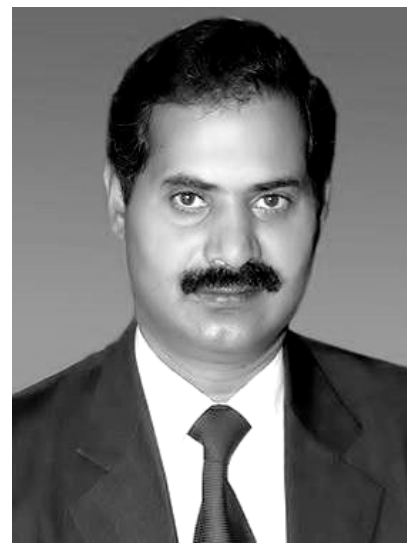

Mubarak Ali graduated from University of the Punjab with B.Sc. (Phys\& Maths) in 1996 and M.Sc. Materials Science with distinction at Bahauddin Zakariya University, Multan, Pakistan (1998); thesis work completed at Quaid-i-Azam University Islamabad. He gained Ph.D. in Mechanical Engineering from Universiti Teknologi Malaysia under the award of Malaysian Technical Cooperation Programme (MTCP;2004-07) and postdoc in advanced surface technologies at Istanbul Technical University under the foreign fellowship of The Scientific and Technological Research Council of Turkey (TÜBITAK; 2010). He completed another postdoc in the field of nanotechnology at Tamkang University Taipei (2013-2014) sponsored by National Science Council now M/o Science and Technology, Taiwan (R.O.C.). Presently, he is working as Assistant Professor on tenure track at COMSATS Institute of Information Technology, Islamabad campus, Pakistan (since May 2008) and prior to that worked as assistant director/deputy director at M/o Science \& Technology (Pakistan Council of Renewable Energy Technologies, Islamabad; 2000-2008). He was invited by Institute for Materials Research (IMR), Tohoku University, Japan to deliver scientific talk on growth of synthetic diamond without seeding treatment and synthesis of tantalum carbide. He gave several scientific talks in various countries. His core area of research includes materials science, physics \& nanotechnology. He was also offered the merit scholarship (for PhD study) by the Government of Pakistan but he couldn't avail. He is author of several articles published in various periodicals (https://scholar.google.com.pk/citations?hl=en\&user=UYjvhDwAAAAJ). 